\title{
A comparative study on changes in the use of heat-not-burn tobacco products based on whether apartment buildings have designated non-smoking areas
}

\author{
Jieun Hwang', Sung-il Cho ${ }^{1,2}$
}

\begin{abstract}
INTRODUCTION Since 2016, multi-family housing developments in South Korea can designate public areas such as staircases, elevator, corridors, and underground parking lots as non-smoking areas if at least half of the households residing in the development agree. This study investigated whether there were changes in the use of heat-not-burn tobacco products $(\mathrm{HnB})$ based on whether non-smoking areas were introduced in multi-family housing developments.

METHODS An online survey of 1200 apartment residents (599 men and 601 women) in seven metropolitan cities in South Korea was conducted from 10 to 18 October 2018.

RESULTS Among the 1200 people who completed the survey, 493 were smokers (351 men and 142 women), of whom 287 (195 men and 92 women) were currently using HnB. In total, $51.5 \%(n=148)$ of the HnB users reported that their smoking frequency inside $(n=75)$ or outside $(n=73)$ the apartment building increased after using $\mathrm{HnB}$, whereas the smoking frequency of $27.5 \%(n=79)$ decreased and that of $20.9 \%(n=6)$ remained unchanged. Of the HnB users, $25.4 \%(n=73)$ were currently living in non-smoking apartments, of whom $39.7 \%$ reported that the smoking frequency outside the apartment building increased. On the other hand, of 214 people who did not currently reside in non-smoking apartments, $30.4 \%$ reported that the smoking frequency in the apartment increased.

CONCLUSIONS For smokers to quit smoking, the expansion of non-smoking areas should be accompanied by the facilitation of a smoke-free atmosphere and a smoking-cessation service for smokers.
\end{abstract}

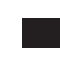

\begin{abstract}
AFFILIATION
1 College of Health Science, Dankook University, Cheonan, Republic of Korea

2 Department of Public Health Science, Graduate School of Public Health, Seoul National University, Seoul, Republic of Korea
\end{abstract}

\section{CORRESPONDENCE TO}

Sung-il Cho. Department of Public Health Science, Graduate School of Public Health, and Institute of Health and Environment, Seoul National University, 1 Gwanak-ro,

Gwanak-gu, Seoul, 08826, Republic of Korea. E-mail: persontime@hotmail.com ORCID ID: https://orcid.org/0000-00034085-1494

\section{KEYWORDS}

heat-not-burn ( $\mathrm{HnB})$, non-smoking areas, multi-family housing,

smoking, tobacco

Received: 24 August 2020

Revised: 13 April 2021

Accepted: 22 April 2021

\section{INTRODUCTION}

In terms of regulating non-smoking areas, indoor public spaces take priority over personal spaces. The World Health Organization Framework Convention on Tobacco Control (FCTC) recommends that countries should designate public spaces such as indoor workplaces and public transportation facilities as non-smoking areas to protect non-smokers from exposure to cigarette smoke. However, although the FCTC encourages the allocation of personal spaces such as personal vehicles and offices as non-smoking areas, it does not make any recommendations on multi-family housing.

Besides the apartment units themselves, indoor staircases, corridors, underground parking lots, and elevators in apartment buildings are shared among the residents. For this reason, smoking in shared areas not only deteriorates indoor air quality but also exposes the non-smoking population to secondhand smoke.

In South Korea, a large proportion of the population lives in apartment buildings, and smoking in common areas is one of the major causes of conflict among residents. To address this problem, 
the South Korean government issued a revision to the National Health Promotion Act in 2016, stating that if at least half the residents of an apartment building agree, hallways, staircases, elevators and underground parking lots can be entirely or partially designated as non-smoking areas. Upon the designation of common areas in multi-family housing developments as non-smoking areas based on a residential vote, instances of smoking in these areas can lead to fines of 50000 won (about US\$ 4.5). This also applies to non-smoking areas designated in indoor public spaces such as hospitals, schools and public transportation.

Designating spaces as non-smoking areas affects the smoking behavior of smokers. The designation of large non-smoking areas in workplaces and public spaces can provide momentum for smokers to quit smoking, leading to a decline in smoking prevalence and tobacco product consumption ${ }^{1}$. However, whether the designation of common residential spaces as non-smoking areas influences smoking behavior at residence remains unknown ${ }^{2,3}$.

Recently, tobacco users have switched from using combusted cigarettes to heat-not-burn tobacco products $(\mathrm{HnB})$ for various reasons. Users consider HnB less harmful compared to combusted cigarettes and think that their use can help with smoking cessation ${ }^{4}$. In contrast to combusted cigarettes, $\mathrm{HnB}$ are more frequently used in indoor spaces because they produce less odour ${ }^{5}$.

Although $\mathrm{HnB}$ sales are prohibited in some countries, global sales have continued to rise. As of 2018, the global market for $\mathrm{HnB}$ was expected to be worth US\$4.4 billion ${ }^{3}$. Euromonitor forecasted that, although the combusted cigarette market will lose US $\$ 7.7$ billion by 2021 , the HnB market will expand by up to US\$13.2 billion during the same period ${ }^{6}$. Since June 2017, after Philip Morris International, British American Tobacco, and KT\&G released the products IQOS, glo, and lil, respectively, in South Korea, competition among $\mathrm{HnB}$ producers has intensified nationally. HnB sales in Korea have been steadily increasing since this product was released, faster than in any other country in the world ${ }^{7}$.

Therefore, this study examined whether the designation of non-smoking areas in multi-family housing developments leads to changes in smoking behavior; namely, where smoking occurs and smoking frequency. Because $\mathrm{HnB}$ use is increasing in South Korea, the study focused on HnB users.

\section{METHODS}

\section{Sampling protocol}

In this study, we conducted an online survey, recruiting adults who had been living in an apartment for more than 6 months. Surveys were conducted using the quota sampling method while considering ages and regions. We aimed to recruit 1200 people in total, limiting the geographical scope to seven metropolitan cities (Seoul, Busan, Daegu, Incheon, Gwangju, Daejeon, and Ulsan). Surveys were conducted by a professional research institute as online self-administered questionnaires. To recruit participants, the company sent a survey participation notice to an online panel administered by the company. Applicants could participate in the survey after consenting to the study. Each participant received 5000 won (about US\$4.5) mobile gift card for completing the survey. Surveys were conducted from 10 to 18 October 2018.

\section{Measures}

The subjects were asked what kinds of cigarette/ tobacco products they had used in their entire life, i.e. cigarettes, cigars, snus, hookah or waterpipe and/ or $\mathrm{HnB}$ that produce aerosols containing nicotine and other chemicals using battery powered heatingsystems such as IQOS, glo, and lil, and whether they were still using this product ${ }^{7}$. We also surveyed $\mathrm{HnB}$ users regarding changes in the amount of smoking inside and outside the apartment after using an $\mathrm{HnB}$, whether smoking frequency increased inside or outside the apartment building, whether overall smoking frequency decreased, or whether no changes occurred. In addition, the subjects were asked whether the building they currently lived in designated common areas such as hallways, staircases, elevators and underground parking lots entirely or partially as nonsmoking areas. Residents who provided 'designated' as an answer were classified as living in non-smoking multi-family housing; those who answered 'not designated' or 'not known' were classified as residents of multi-family housing without non-smoking areas.

\section{Statistical analysis}

A frequency analysis was conducted to assess the 
number and percentage of subjects according to demographic characteristics and change after the usage of $\mathrm{HnB}$ depending on designation of nonsmoking apartment buildings. In addition, crude logistic regression was applied to explore the relationship between smoking pattern and residential type among heat-not-burn tobacco products. The results are presented as odds ratios (ORs) and 95\% confidence interval (CIs). Data were analyzed using
SPSS ver. 25 software (IBM Corporation, Armonk, NY, USA), and $\mathrm{p}<0.05$ was considered statistically significant.

\section{RESULTS}

Of all respondents, $49.9 \%(n=599)$ were male and $50.1 \%(\mathrm{n}=601)$ were female (Table 1$)$. Respondents in their 40s made up the largest age group, and the largest proportion of respondents lived in Seoul

Table 1. Demographic characteristics and change after the usage of heat-not-burn tobacco products, depending on designation of non-smoking apartment buildings in South Korea $2018(\mathrm{~N}=1200)$

\begin{tabular}{|c|c|c|c|}
\hline Characteristics & $\begin{array}{l}\text { Total } \\
n(\%)\end{array}$ & $\begin{array}{l}\text { Residents in designated } \\
\text { non-smoking apartments } \\
(n=243) \\
n(\%)\end{array}$ & $\begin{array}{l}\text { Residents in unspecified } \\
\text { non-smoking apartments } \\
(n=957) \\
n(\%)\end{array}$ \\
\hline \multicolumn{4}{|l|}{ Gender } \\
\hline Male & 599 (49.9) & $129(53.1)$ & $470(49.1)$ \\
\hline Female & $601(50.1)$ & $114(46.9)$ & 487 (50.9) \\
\hline \multicolumn{4}{|l|}{ Age (years) } \\
\hline 20-29 & 231 (19.3) & $55(22.6)$ & $176(18.4)$ \\
\hline 30-39 & $242(20.2)$ & 45 (18.5) & $197(20.6)$ \\
\hline $40-49$ & 271 (22.5) & 63 (25.9) & $208(21.7)$ \\
\hline $50-59$ & $270(22.5)$ & $49(20.2)$ & $221(23.1)$ \\
\hline$\geq 60$ & 186 (15.5) & 31 (12.8) & 155 (16.2) \\
\hline \multicolumn{4}{|l|}{ Region } \\
\hline Seoul & 527 (43.9) & 127 (52.3) & $400(41.8)$ \\
\hline Busan & 180 (15.0) & 32 (13.2) & $148(15.5)$ \\
\hline Daegu & 129 (10.8) & 19 (7.8) & $110(11.5)$ \\
\hline Incheon & 154 (12.8) & 30 (12.3) & $124(13.0)$ \\
\hline Gwangju & $73(6.1)$ & $10(4.1)$ & $63(6.6)$ \\
\hline Daejeon & 77 (6.4) & $16(6.6)$ & $61(6.4)$ \\
\hline Ulsan & $60(5.0)$ & $9(3.7)$ & $51(5.2)$ \\
\hline \multicolumn{4}{|l|}{ Cigarette use experience on entire life ${ }^{1}$} \\
\hline Cigarettes & $633(52.8)$ & $125(51.4)$ & $508(53.1)$ \\
\hline E-cigarettes & $348(29.0)$ & $82(33.7)$ & $266(27.8)$ \\
\hline Heat-not-burn tobacco products & 287 (23.9) & $73(30.0)$ & $214(22.4)$ \\
\hline Cigars & $239(19.9)$ & $48(19.8)$ & $191(20.0)$ \\
\hline Snus & $122(10.2)$ & $28(11.5)$ & $94(9.8)$ \\
\hline Hookah or waterpipe & $74(6.2)$ & $20(8.2)$ & $54(5.6)$ \\
\hline \multicolumn{4}{|l|}{ Smoking pattern change ${ }^{2}$} \\
\hline $\begin{array}{l}\text { Increase in smoking frequency outside the } \\
\text { apartment building }\end{array}$ & $73(25.5)$ & $29(39.7)$ & $44(20.6)$ \\
\hline Increase in smoking frequency inside the apartment & $75(26.1)$ & $10(13.7)$ & $65(30.4)$ \\
\hline Decrease in smoking frequency & $79(27.5)$ & $17(23.3)$ & $62(29.0)$ \\
\hline No change & $60(20.9)$ & $17(23.3)$ & $43(20.0)$ \\
\hline
\end{tabular}

1 Multiple answers. 2 For heat-not-burn tobacco products users. 
Figure 1. The relationship between smoking pattern and residential type among heat-not-burn tobacco products users ( $\mathbf{N}=287)$

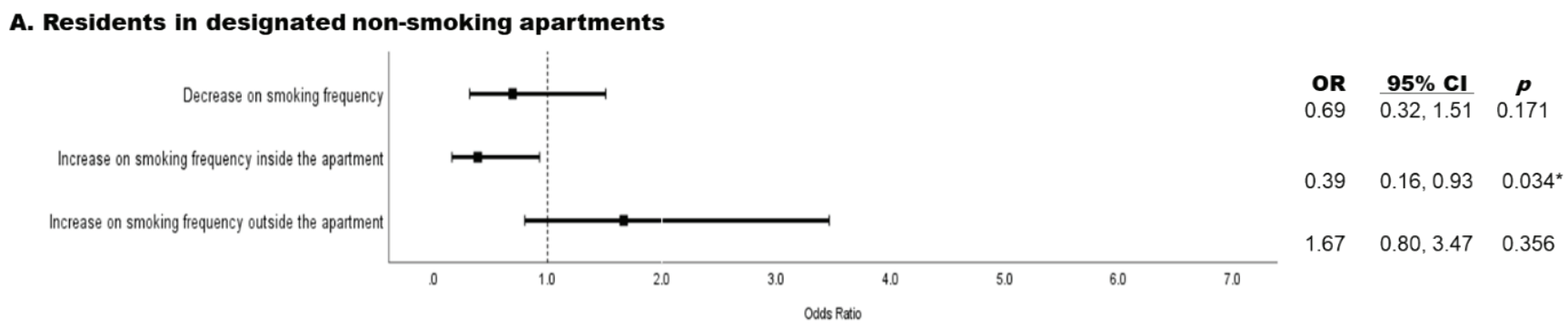

B. Residents in unspecified non-smoking apartments

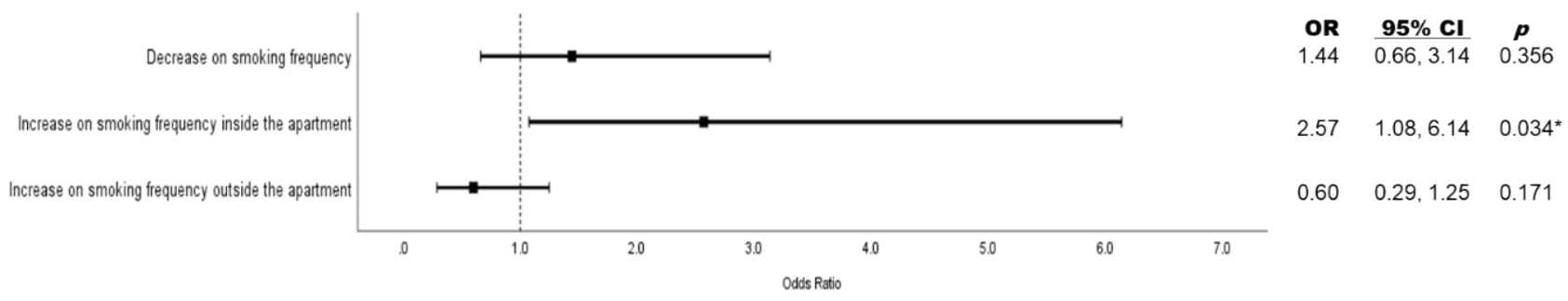

${ }^{*} \mathrm{OR}$ : odds ratio. Cl: confidence interval. Crude logistic regression was applied to explore the relationship between smoking pattern (Reference: no change) for residents depending on designation of non-smoking apartment buildings among heat-not-burn tobacco products users.

$(43.9 \%)$. In terms of non-smoking designations, $20.3 \%(n=243)$ of the respondents were living in apartment buildings with non-smoking common areas, whereas $69.7 \%(\mathrm{n}=957)$ lived in buildings without such designated areas. More than half the respondents $(52.8 \%)$ had used cigarettes or other tobacco products at least once in their life, and $29.0 \%$ had used e-cigarettes.

Of the 1200 respondents, 287 (23.9\%) had used HnB. Of these 287 people, 73 people were currently living in buildings with designated non-smoking areas, and 214 were not. In terms of changes in the frequency of smoking by $\mathrm{HnB}$ users, respondents $(\mathrm{n}=79 ; 27.5 \%)$ who reported a decrease in smoking amount inside or outside the apartment comprised the largest proportion, followed by those who reported an increase in smoking inside $(n=75$; $26.1 \%)$ the apartment and outside $(n=73 ; 25.5 \%)$ the apartment building, whereas $20.9 \%(n=60)$ did not report any changes.

In terms of changes in where smoking took place and the smoking frequency, residents of nonsmoking apartment buildings who reported an increase in smoking outside the apartment building comprised the largest group of respondents (39.7\%). Residents of buildings without designated non- smoking areas who reported an increase in smoking at the apartment comprised the next largest group of respondents $(30.4 \%)$. This difference in proportions was statistically significant (Pearson $\chi^{2}$ test; $\mathrm{p}=0.002)$.

Figure 1 shows the results of a crude logistic regression analysis of the relationship between smoking pattern change and residential type among HnB users. A residential type was significantly associated with smoking frequency inside the apartment building. Among $\mathrm{HnB}$ users in designated non-smoking apartments, the probability of increase in smoking frequency inside was lower than for users whose usage patterns have not changed (increase in smoking frequency inside the apartment: $\mathrm{OR}=0.39$; 95\% CI: $0.16-0.93, p=0.034)$. The HnB users living in unspecified non-smoking apartments were more likely to increase smoking frequency inside compared to those who did not change pattern (increase in smoking frequency inside the apartment: $\mathrm{OR}=2.57$; 95\% CI: 1.08-6.14).

\section{DISCUSSION}

Since HnB started being sold in South Korea, the number of $\mathrm{HnB}$ users has increased steadily. A month after IQOS was released in South Korea (September 
2017 ), only $5.7 \%$ of users had experience with this product. However, in October 2018 when this survey was conducted and when IQOS, lil, and glo were all available in the market, $23.9 \%$ people reported that they had used $\mathrm{HnB}$, indicating that the consumption of these products had increased. Although e-cigarettes have been available in South Korea since $2007^{8}$, it is noteworthy that similar proportions of people reported experience with using e-cigarettes and $\mathrm{HnB}$. This implies that HnB products, although a novel product, are rapidly dominating the Korean tobacco market.

HnB companies advertise their wares as state-ofthe-art products representing new technological innovations to a target audience that includes youth $^{9,10}$. Mainly, these companies claim that, unlike existing tobacco products, $\mathrm{HnB}$ products are harmless to the human body, and this marketing strategy is rather successful. As the results of the survey showed, after they started using these products, $\mathrm{HnB}$ users increased rather than decreased the amount of smoking inside the apartment and outside the building. It was assumed that $\mathrm{HnB}$ produced less odor than existing tobacco products ${ }^{5}$, thus facilitating their use inside and outside the apartment. However, the safety of smoking $\mathrm{HnB}$ has been not sufficiently clarified. Because the smoke from $\mathrm{HnB}$ may contain carcinogenic chemicals, it can adversely affect indoor air quality and the health of people exposed to secondhand smoke ${ }^{11,12}$. Therefore, accurate and readily available public health information as well as appropriate regulations are needed to prevent the indoor use of $\mathrm{HnB}$ and discourage the initial use of these products.

A new finding in this study was that $\mathrm{HnB}$ users changed their smoking behavior according to the characteristics of their residence. The designation of non-smoking common areas does not forbid the act of smoking at the apartment, only in common areas in apartment buildings. Smokers who lived in non-smoking apartment buildings tended to smoke outside instead of inside their residences. These results suggest that smokers could change their smoking location due to the creation of smokefree environments, such as by designation as nonsmoking areas.

\section{CONCLUSIONS}

Although $\mathrm{HnB}$ use and the availability of non-smoking areas can influence the amount of smoking and smoking location of residents of multi-family housing, these changes do not necessarily lead to smoking cessation. In particular, a significant proportion of respondents reported an increase in smoking amount despite the expansion of non-smoking areas in the apartment buildings. Thus, the designation of such areas without a plan for smoking cessation might cause the impacts from secondhand smoke to be moved to another location. In addition to designating smoke-free areas, comprehensive approaches to encourage smokers to quit smoking, such as providing appropriate anti-smoking support services and developing effective messaging for smoking cessation, are needed.

\section{REFERENCES}

1. Fichtenberg CM, Glantz SA. Effect of smoke-free workplaces on smoking behaviour: systematic review. BMJ. 2002;325(7357):188. doi:10.1136/bmj.325.7357.188

2. Ye X, Chen S, Yao Z, et al. Smoking behaviors before and after implementation of a smoke-free legislation in Guangzhou, China. BMC Public Health. 2015;15:982. doi:10.1186/s12889-015-2353-6

3. Heated Tobacco Products Market Size, Share \& Trends Analysis Report By Product (Stick, Leaf), By Distribution Channel (Online, Offline), By Region, And Segment Forecasts, 2019 - 2025. Grand View Research. July, 2019. Accessed April 13, 2021. https://www.grandviewresearch. com/industry-analysis/heated-tobacco-products-htpsmarket

4. Fung MDT, Diemert L, Zhang B, O'Connor SC, Schwartz R. Awareness and Perceived Risk of Heated Tobacco Products. Tob Regul Sci. 2020;6(1):15-19. doi:10.18001/TRS.6.1.2

5. Cho H, Kim H, Lee C, et al. Analysis of Heated Tobacco Products Usage and Its Effect on Attempts to Smoking Cessation. Report in Korean. Ministry of Health and Welfare, University of Ulsan; 2018.

6. Cigarettes to Record US\$7.7 Billion Loss by 2021 as Heated Tobacco Grows 691 Percent. Euromonitor International. June 24, 2017. Accessed April 13, 2021. https://blog.euromonitor.com/cigarettes-record-lossheated-tobacco-grows-691-percent/

7. Tobacco control fact sheet: Domestic market trend of heated tobacco products. Report in Korean. Korea Health Promotion Institute; 2019. Accessed April 13, 2021. https://nosmk.khealth.or.kr/nsk/ ezpdfwebviewer/ezpdf/customLayout.jsp?contentId=/ site/ntcc/upload/infoManagement/20190821155033_ ab817c9349cf9c4f6877e1894a1faa00.pdf

8. Kim J, Yu H, Lee S, Paek YJ. Awareness, experience and prevalence of heated tobacco product, IQOS, among 
young Korean adults. Tob Control. 2018;27(Suppl 1):s74-s77. doi:10.1136/tobaccocontrol-2018-054390

9. McKelvey K, Popova L, Kim M, et al. Heated tobacco products likely appeal to adolescents and young adults. Tob Control. 2018;27(Suppl 1):s41-s47. doi:10.1136/tobaccocontrol-2018-054596

10. Hair EC, Bennett M, Sheen E, et al. Examining perceptions about IQOS heated tobacco product: consumer studies in Japan and Switzerland. Tob Control. 2018;27(Suppl 1):s70-s73. doi:10.1136/tobaccocontrol-2018-054322

11. Cancelada L, Sleiman M, Tang X, et al. Heated Tobacco Products: Volatile Emissions and Their Predicted Impact on Indoor Air Quality. Environ Sci Technol. 2019;53(13):7866-7876. doi:10.1021/acs.est.9b02544

12. Meišutovič-Akhtarieva M, Prasauskas T, Čiužas D, et al. Impacts of exhaled aerosol from the usage of the tobacco heating system to indoor air quality: A chamber study. Chemosphere. 2019;223:474-482. doi:10.1016/j.chemosphere.2019.02.095
ACKNOWLEDGMENTS

The authors thank the Korea Health Promotion Institute.

CONFLICTS OF INTEREST

The authors have completed and submitted the ICMJE Form for Disclosure of Potential Conflicts of Interest and none was reported.

\section{FUNDING}

This study was funded by the Korea Health Promotion Institute.

ETHICAL APPROVAL AND INFORMED CONSENT

This study was approved by the Institutional Review Board of the Seoul National University (No. 1809/003-007). Applicants could participate in the survey after consenting to the study.

\section{DATA AVAILABILITY}

The data supporting this research cannot be made available for privacy reasons.

\section{AUTHORS' CONTRIBUTIONS}

$\mathrm{JH}$ designed the study, analyzed and interpreted the data, and wrote the manuscript. SC contributed to the study design and interpretation of the results.

PROVENANCE AND PEER REVIEW

Not commissioned; externally peer reviewed. 